\title{
Semiconducting polymer-buckminsterfullerene heterojunctions: Diodes, photodiodes, and photovoltaic cells
}

\author{
N. S. Sariciftci, D. Braun, and C. Zhang \\ Institute for Polymers and Organic Solids, University of California at Santa Barbara, Santa Barbara, \\ California 93106-5090 \\ V. I. Srdanov \\ Center for Quantized Electronic Structures, University of California at Santa Barbara, Santa Barbara, \\ California 93106-5090
}

\author{
A. J. Heeger, G. Stucky, and F. Wudl \\ Institute for Polymers and Organic Solids, University of California at Santa Barbara, Santa Barbara, \\ California 93106-5090
}

\begin{abstract}
The characterization of rectifying heterojunctions (diodes) fabricated from a semiconducting polymer, a soluble derivative of poly (phenylene-vinylene), and buckminsterfullerene, $\mathrm{C}_{60}$, are reported. Rectification ratios in the current versus voltage characteristics exceed $10^{4}$. When illuminated, the devices exhibit a large photoresponse as a result of photoinduced electron transfer across the heterojunction interface from the semiconducting polymer (donor) onto $\mathrm{C}_{60}$ (acceptor). The photodiode and photovoltaic responses are characterized. Photoinduced electron transfer across the donor-accepted rectifying heterojunction offers potential for photodetector and for solar cell applications.
\end{abstract}

Photoinduced electron transfer with a subpicosecond transfer rate has been demonstrated in composites of the semiconducting polymer, poly[2-methoxy,5-(2'-ethylhexyloxy)-1,4-phenylene-vinylene], MEH-PPV, and buckminsterfullerene, $\mathrm{C}_{60}{ }^{1,2}$ The observation of photoinduced electron transfer between the donor, MEH-PPV, and the acceptor, $\mathrm{C}_{60}$, suggests the possibility of utilizing this phenomenon at the interface between MEH-PPV and $\mathrm{C}_{60}$ in a thin film heterojunction bilayer.

In this letter, we report the fabrication and characterization of heterojunction diodes fabricated from MEHPPV and $\mathrm{C}_{60}$. Rectification ratios in excess of $10^{4}$ are obtained. A large photoresponse is observed in the current vs voltage characteristics, resulting from photoinduced electron transfer across the interface.

The utility of MEH-PPV as a processible polymer ${ }^{3-5}$ with the electronic and optical properties of a semiconductor has already been demonstrated in Schottky-type light emitting devices which exhibit relatively high efficiency electroluminescence. ${ }^{6,7}$ MEH-PPV is a member of the family of conjugated polymers; these quasi-one-dimensional conducting polymers have relatively broad $\pi$ (valence) and $\pi^{*}$ (conduction) bands, and they can be doped, with resulting properties that span the full range from insulator to metal. ${ }^{8} \mathrm{MEH}-\mathrm{PPV}$ is a weak donor (as are many other conjugated polymers) which can be oxidized with relative ease. ${ }^{9}$ Buckminsterfullerene has been demonstrated to be an $n$-type semiconductor which acts as a relatively strong acceptor. ${ }^{10,11}$

Comparison of the energies of the lowest unoccupied states of $\mathrm{C}_{60}$ with the energies of the highest occupied states of semiconducting polymers suggests that partial charge transfer can occur in the ground state. ${ }^{12}$ Studies of the MEH-PPV/ $\mathrm{C}_{60}$ composites have shown, however, that although charge transfer between the donor and the accep- tor does not occur in the ground state, charge separation can be photoinduced with high efficiency. ${ }^{1,2}$

The heterojunction diodes consist of successive layers of MEH-PPV, $\mathrm{C}_{60}$, and gold deposited onto indium/tinoxide (ITO) coated glass substrates. The MEH-PPV layer was spin-cast onto the ITO-glass substrate from $1 \%$ xylene solution at $2000 \mathrm{rpm}$. The $\mathrm{C}_{60}$ was subsequently vacuum evaporated to form the heterojunction. Typical thicknesses are $1000 \AA$ for both the MEH-PPV and the $\mathrm{C}_{60}$. The metal (gold or aluminum) contacts were deposited on top of the devices by vacuum evaporation at pressures below $1 \times 10^{-6}$ Torr and patterned with a shadow mask to define active areas of $0.1 \mathrm{~cm}^{2}$. All processing steps are carried out in nitrogen atmosphere or under vacuum, except the transfers to and from the vacuum chamber for $\mathrm{C}_{60}$ deposition.

Electrical data were obtained with a Keithley 236 Source-Measure Unit. The $514.5 \mathrm{~nm}$ line of an argon ion laser was used as the light source in measurements of the intensity dependence of the photocurrent. A tungsten lamp with a single-grating monochromator was used for the photoresponse measurement. The light was mechanically chopped at the exit slit and a lock-in amplifier was used to detect the photocurrent. All spectra have been corrected for the spectral response of the lamp and monochromator by normalization to the response of a calibrated silicon photodiode.

Figure 1 shows the current-voltage characteristics of the heterojunction device consisting of ITO/MEH-PPV/ $\mathrm{C}_{60} / \mathrm{Au}$. Positive bias is defined as positive voltage applied to the ITO contact. Exponential turn-on up to $500 \mathrm{mV}$ in forward bias is clearly observable; the rectification ratio is approximately $10^{4}$.

In order to test if the ITO or the gold electrodes form blocking (or rectifying) contacts, the following three layer devices were prepared: gold/MEH-PPV/gold, gold/MEH- 


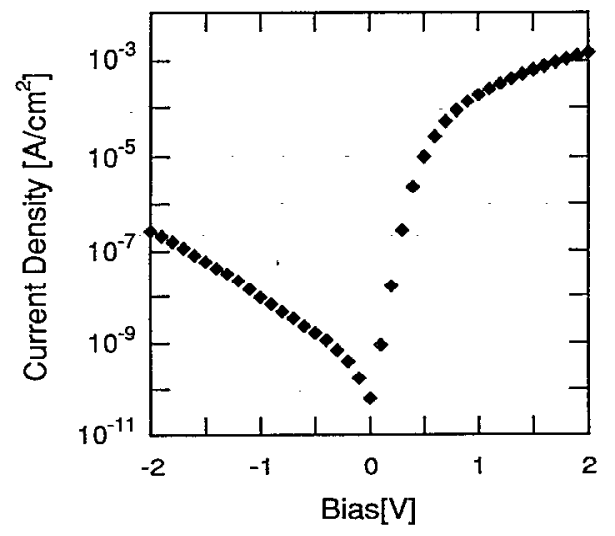

FIG. 1. Dark current vs voltage characteristics of the ITO/MEH-PPV/ $\mathrm{C}_{60} / \mathrm{Au}$ device at room temperature.

PPV/ITO, gold $/ \mathrm{C}_{60} /$ gold, and gold $/ \mathrm{C}_{60} /$ ITO. since all such devices have completely symmetric and linear current-voltage characteristics, we conclude that gold and ITO form non-rectifying contacts both to MEH-PPV and to $\mathrm{C}_{60}$, in agreement with the reported absence of rectification in $\mathrm{Au} / \mathrm{C}_{60} / \mathrm{ITO}$ devices. ${ }^{13} \mathrm{We}$, therefore, attribute the rectifying behavior of the four layer device to the interface of the heterojunction between the semiconducting polymer and $\mathrm{C}_{60}$.

The current-voltage characteristic of the device changes dramatically upon illumination with visible light. Figure 2 shows the current-voltage data, with the ITO/ $\mathrm{MEH}-\mathrm{PPV} / \mathrm{C}_{60} / \mathrm{Au}$ device in the dark and with the device illuminated with $514.5 \mathrm{~nm}$ light with intensity $\left(P_{\text {in }}\right)$ of $\approx 1$ $\mathrm{mW} / \mathrm{cm}^{2}$. The open circuil voltage $\left(V_{\text {oc }}\right)$ is $0.44 \mathrm{~V}$ which saturates to around $0.53 \mathrm{~V}$ under stronger illumination. The short circuit current density $\left(J_{\mathrm{sc}}\right)$ is $2.08 \times 10^{-6}$ $\mathrm{A} / \mathrm{cm}^{2}$, and the fill factor (FF) can be obtained from the relation

$$
\mathrm{FF}=\frac{\int_{0}^{V_{\mathrm{oc}} J d V}}{J_{\mathrm{sc}} \overline{V_{\mathrm{oc}}}}
$$

The data of Fig. 2 give a fill factor of 0.48 and a power

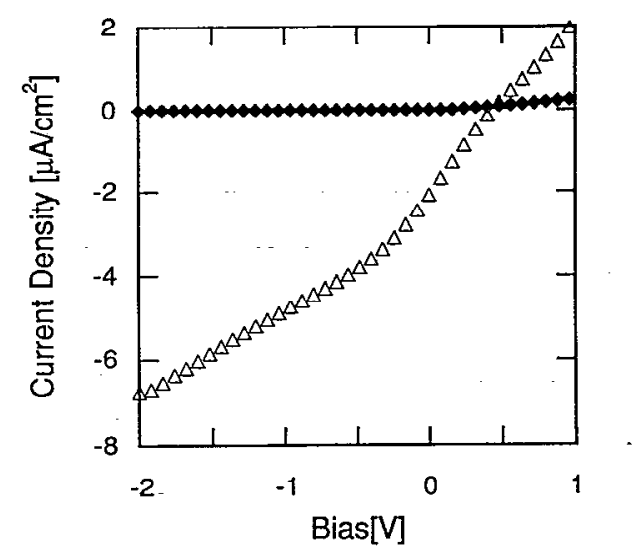

FIG. 2. Current vs voltage characteristics of the ITO/MEH-PPV/ $\mathrm{C}_{60} / \mathrm{Au}$ device in the dark (diamonds) and upon illumination with the $514.5 \mathrm{~nm}$ line from an argon ion laser of $\approx 1 \mathrm{~mW} / \mathrm{cm}^{2}$ (triangles).

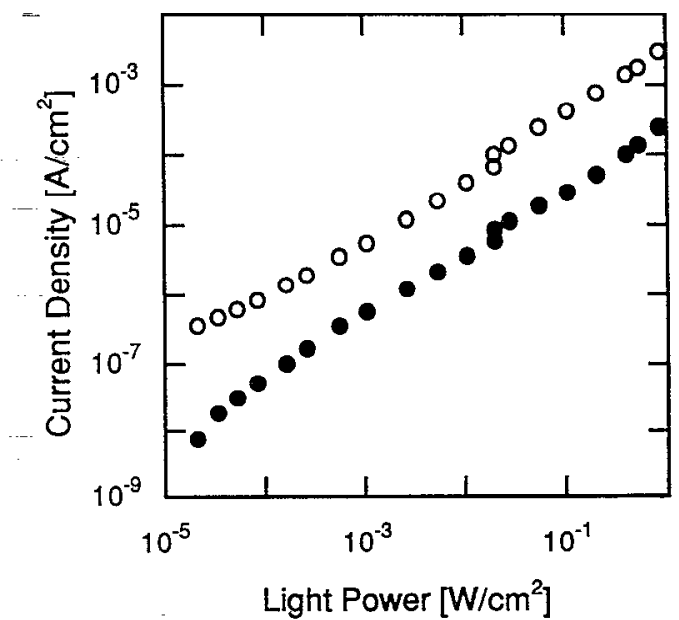

FIG. 3. Short circuit current (closed circles) and photocurrent at $-1 \mathrm{~V}$ bias (open circles) as a function of light intensity for the ITO/MEH$\mathrm{PPV} / \mathrm{C}_{60} / \mathrm{Au}$ device.

conversion efficiency of $0.04 \% .{ }^{14}$ An open circuit voltage of about $0.5 \mathrm{~V}$ appears to be characteristic of the MEH$\mathrm{PPV} / \mathrm{C}_{60}$ interface. Similar values were obtained with ITO/MEH-PPV/ $\mathrm{C}_{60} / \mathrm{Al}$ devices and with $\mathrm{Al} / \mathrm{MEH}-\mathrm{PPV} /$ $\mathrm{C}_{60} / \mathrm{ITO}$ devices. ${ }^{15}$

As expected from the observation of fast photoinduced electron transfer from MEH-PPV to $\mathrm{C}_{60}, 1,2$ a remarkable increase in both forward and reverse bias current is observed to result from photoinduced charge separation at the heterojunction interface. The jump in the current by nearly four orders of magnitude (from $1 \times 10^{-9}$ to $6 \times 10^{-6} \mathrm{~A} / \mathrm{cm}^{2}$ ) upon illumination at $-1 \mathrm{~V}$ (reverse) bias demonstrates that the heterojunction serves as a relatively sensitive photodiode.

Figure 3 shows the dependence of the short curcuit current and the photocurrent at $-1 \mathrm{~V}$ (reversc) bias as a function of the illumination intensity $(514.5 \mathrm{~nm})$. The data in Fig. 3 show no indication of saturation at light intensities up to approximately $1 \mathrm{~W} / \mathrm{cm}^{2}$; i.e., one order of magnitude greater than the terrrestrial solar intensity.

The spectral dependence of the photoresponse of these heterojunction devices is displayed in Fig. 4. The onset of

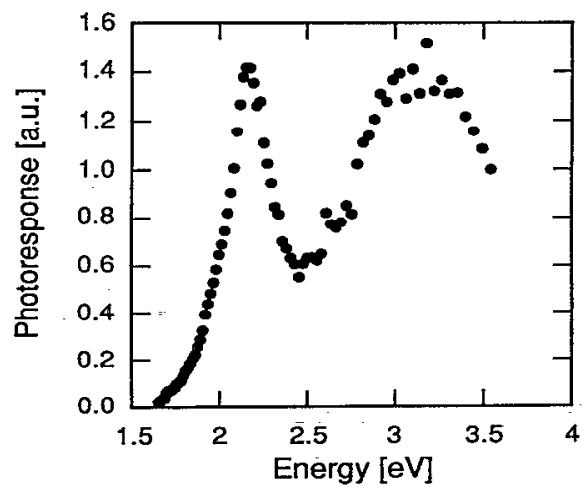

FIG. 4. Spectral response of the photocurrent in ITO/MEH-PPV/ $\mathrm{C}_{60} / \mathrm{Au}$ photodiode at (reverse) $-1 \mathrm{~V}$ bias. 
photocurrent at $\hbar \omega \approx 1.7 \mathrm{eV}$ follows the absorption of MEH-PPV, which initiates the photoinduced electron transfer; ${ }^{1,2}$ note that illumination is from the ITO/MEHPPV side of the device. The minimum in the photocurrent at $\hbar \omega \approx 2.5 \mathrm{eV}$ corresponds to the energy of maximum absorption of MEH-PPV. We propose, therefore, that the MEH-PPV layer acts as a filter which reduces the number of photons reaching the MEH-PPV/ $\mathrm{C}_{60}$ interface. This implies that diffusion of charge carriers in these devices is limited; the photoactive region is restricted to a thin layer adjacent to the interface between the MEH-PPV and $\mathrm{C}_{60}$ layers. Assuming a charge carrier mobility of $\sim 10^{-4} \mathrm{~cm}^{2} /$ $\mathrm{Vs}$, with an applied field of $10^{5} \mathrm{~V} / \mathrm{cm}$ and with a photoinduced carrier lifetime $\sim 1 \mathrm{~ns}^{16}$, the maximum distance a carrier could travel in the MEH-PPV is estimated to be only a few angstroms. Thus, the generation of photoexcitations which result in separated charge carriers occurs at the heterojunction interface.

The demonstration of a rectifying heterojunction between a semiconducting polymer and buckminsterfullerene has potentially important scientific and technological implications. The heterojunction is quite sensitive as a photodetcctor with a dark current below $100 \mathrm{pA} / \mathrm{cm}^{2}$. Moreover, there is potential for using such devices in photovoltaic energy conversion. To be efficient, however, one must use a semiconducting polymer with band gap well-matched to the solar spectrum (for example polythienylenevinylene ${ }^{17}$ ), and one must greatly increase the effective area of the heterojunction interface (e.g., by roughening as, for example, in etched aluminum capacitors). The solubility of semiconducting polymers and of buckminsterfullerene polymers ${ }^{18}$ will facilitate device fabrication with minimal thermal budget and will enable the assembly of mechanically flexible devices, as already been realized with light-emitting polymer diodes. ${ }^{19}$

This work is supported by the Office of Naval Research (ONR Contract No. N00014-91-J-1235) and the Electric Power Research Institute (EPRI Contract No. RP800721). V.I.S. acknowledges the support by the NSF Quantized Electronic Structures Science and Technology Center (QUEST) at UCSB.
${ }^{1}$ N. S. Sariciftci, L. Smilowitz, A. J. Heeger, and F. Wudl, Science (to be published).

${ }^{2}$ N. S. Sariciftci, L. Smilowitz, D. Braun, G. Srdanov, V. Srdanov, F. Wudl, and A. J. Heeger, Synth. Met. (to be published).

${ }^{3}$ F. Wudl, P. M. Allemand, G. Srdanov, Z. Ni, and D. McBranch, in Materials for Non-linear Optics, Chemical Perpectives, edited by S. R. Marder, J. E. Sohn, and G. D. Stucky (American Chemical Society, Washington, DC, 1991), p. 683.

${ }^{4}$ T. W. Hagler, K. Pakbaz, K. F. Voss, and A. J. Heeger, Phys. Rev. B 44, 8652 (1991).

${ }^{5}$ L. Smilowitz and A. J. Heeger, Synth. Met. 48, 193 (1992).

${ }^{6}$ J. H. Burroughs, D. D. C. Bradley, A. R. Brown, R. N. Marks, K. Mackay, R. H. Friend, P. L. Burns, and A. B. Holmes, Nature 347, 539 (1990).

${ }^{7}$ (a) D. Braun and A. J. Heeger, Appl. Phys. Lett. 58, 1982 (1991). (b) D. Braun, A. J. Heeger, and H. Kroemer, J. Electron. Mater. 20, 945 (1991).

${ }^{8}$ A. J. Heeger, S. Kivelson, J. R. Schrieffer and W. P. Su, Rev. Mod. Phys. 60, 781 (1988).

${ }^{9}$ IIandbook of Conducting Polymer Vol. I, II, edited by T. A. Skotheim (Marcel Dekker, New York, 1986).

${ }^{10}$ B. Miller, J. M. Rosamilia, G. Dabbagh, R. Tycko, R. C. Haddon, A. J. Muller, W. Wilson, D. W. Murphy, and A. F. Hebard, J. Am. Chem. Soc. 113, 6291 (1991).

${ }^{11}$ P. M. Allemand, A. Koch, F. Wudl, Y. Rubin, F. Diederich, M. M. Alvarez, S. J. Anz, and R. L. Whetten, J. Am. Chem. Soc. 113, 1050 (1991).

${ }^{12}$ S. Morita, A. A. Zakhidov, and K. Yoshino, Solid State Commun. 82, 249 (1992).

${ }^{13}$ H. Yonehara and C. Pac, Appl. Phys. Lett. 61, 575 (1992).

${ }^{14} \mathrm{By}$ choosing the definition of the fill factor according to $\mathrm{FF}=J_{m} V_{m}$ ' $J_{s c} V_{o c}$ where $J_{m}$ and $V_{m}$ are voltage and current for maximum power output $\left(J_{m} \approx 0.88 \mu \mathrm{A} / \mathrm{cm}^{2}\right.$ and $V_{m} \approx 0.24 \mathrm{~V}$ ), the fill factor becomes 0.2 and the calculated conversion efficiency is $\eta=0.02 \%\left(\eta=\mathrm{FF} J_{\mathrm{sc}} V_{\mathrm{oc}} / P_{i n}\right.$.

${ }^{15}$ For an Al/MEH-PPV/ITO device without $\mathrm{C}_{60}$, we find that $V_{\text {oc }}$ saturates at $1.4 \mathrm{~V}$, but $J_{\mathrm{sc}}$ is a factor of 20 smaller than in the heterojunction devices. Recently reported $\mathrm{Al} / \mathrm{AlO}_{x} / \mathrm{C}_{60} \mathrm{MIS}$ junctions (see Ref. 13) have a higher photoconversion efficiency, but $J_{\mathrm{sc}}$ and $V_{\mathrm{oc}}$ were not reported.

${ }^{16}$ L. Smilowitz, A. W. Hays, G. Wang, A. J. Heeger, and J. E. Bowers, Synth. Met. (to be published).

${ }^{17}$ S. Yamada, S. Tokito, T. Tsutsui, and S. Saito, J. Chem. Soc. Chem. Commun. 1987, 1448; H. Eckhardt, L. W. Schacklette, K. Y. Jen, and R. L. Elsenbaumer, J. Chem. Phys. 91, 1303 (1989); A. J. Brasset, N. F. Colaneri, D. D. C. Bradley, R. A. Lawrence, R. H. Friend, H. Murata, S. Tokito, T. Tsutsui, and S. Saito, Phys. Rev. B 41, 10586 (1990).

${ }^{18}$ S. Shi, K. C. Khemani, Q. Chan Li, and F. Wudl, J. Am. Chem. Soc. (to be published).

${ }^{19}$ G. Gustafsson, Y. Cao, G. M. Treacy, and F. Klavetter, N. Colaneri, and A. J. Heeger, Nature 357, 477 (1992). 